\title{
Structural, Thermal, Magnetic, and Transport Properties of $\left(\mathrm{La}_{2 / 3} \mathrm{Ca}_{1 / 3}\right)\left(\mathrm{Mn}_{1-x} \mathrm{Sn}_{x}\right) \mathrm{O}_{3-\delta}$ Compounds
}

\author{
J. Przewoźnik ${ }^{a}$, J. Chmist ${ }^{a}$, L. Kolwicz-Chodak ${ }^{a}$,

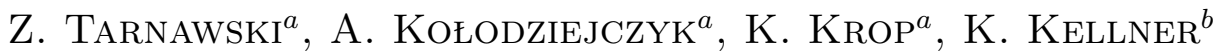 \\ AND G. GRITZNER ${ }^{b}$ \\ ${ }^{a}$ Faculty of Physics and Nuclear Techniques \\ AGH University of Science and Technology \\ al. Mickiewicza 30, 30-059 Cracow, Poland \\ ${ }^{b}$ Institut für Chemische Technologie Anorganischer Stoffe \\ Johannes Kepler Universität Linz, 4040 Linz, Austria
}

The structural, magnetic, and electrical transport properties of Sn-doped manganite $\mathrm{La}_{0.67} \mathrm{Ca}_{0.33} \mathrm{Mn}_{1-x} \mathrm{Sn}_{x} \mathrm{O}_{3-\delta}(x=0,0.01,0.03$, and $\delta \approx 0.06$ ) compounds were studied using X-ray powder diffraction, scanning electron microscopy, AC susceptometer and vibrating sample magnetometer measurements as well as four-probe resistance measurements. The specific heat was measured by the heat-pulse method. The Curie temperature $T_{\mathrm{C}}$ and the metal-insulator transition temperature $T_{\mathrm{M}-\mathrm{I}}$ decreased nonlinearly with increasing Sn content. The $T_{\mathrm{C}}$ and $T_{\mathrm{M}-\mathrm{I}}$ values, for the $x=0,0.01$, and 0.03 compounds were separated by $18.2 \mathrm{~K}, 66.3 \mathrm{~K}$, and $10 \mathrm{~K}$, respectively. The resistivity above $T_{\mathrm{C}}$ for all of these compounds followed the Mott variable-range-hopping model. This allowed the estimation of the localization lengths of $2.2 \AA(x=0), 1.33 \AA(x=0.01)$ and $1.26 \AA(x=0.03)$. The $x=0$ and $x=0.01$ compounds exhibited anomalies of $R(T)$ at corresponding $T_{\mathrm{C}}$ and allowed the separation of the magnitude of the purely magnetic contribution to the resistance which for $x=0$ was $\approx 5.7 \Omega$ and for $x=0.01, \approx 22.4 \Omega$. The specific heat of the Sn-free sample exhibited a sharp peak at $T_{\mathrm{C}}$. With increasing Sn content the peak at $T_{\mathrm{C}}$ broadened and the area under the peak decreased. For $x=0.03$ the peak was hardly detectable. Our results on $\mathrm{La}_{0.67} \mathrm{Ca}_{0.33} \mathrm{Mn}_{1-x} \mathrm{Sn}_{x} \mathrm{O}_{3}$ reveal that a small substitution of $\mathrm{Sn}^{4+}$ for $\mathrm{Mn}^{4+}$ suppresses double exchange interactions and strongly affects the magnetic, thermal, and transport properties of the parent compound.

PACS numbers: 75.47.Lx, 75.47.Gk, 75.40.Cx 


\section{Introduction}

Substitution of $\mathrm{Ca}^{2+}$ for $\mathrm{La}^{3+}$ in the antiferromagnetic insulator $\mathrm{LaMnO}_{3}$ gives rise to an equivalent amount of $\mathrm{Mn}^{4+}$ which induces ferromagnetism (FM) and metallicity in (La,Ca) $\mathrm{MnO}_{3}$. The maximum Curie temperature $T_{\mathrm{C}}$ as well as the minimum resistivity is observed for the $\mathrm{La}_{0.67} \mathrm{Ca}_{0.33} \mathrm{MnO}_{3}$ compound. The colossal magnetoresistance (CMR) is found to appear near the metal-insulator (M-I) transition. This transition is characterized by a peak in the resistivity appearing at the $T_{\mathrm{M}-\mathrm{I}}$ temperature. For single crystals and frequently for polycrystalline compounds this electronic transition is accompanied by simultaneous FM-to-paramagnetic (PM) transition at nearly the same temperature [1]. However, polycrystalline compounds with small grain sizes often show big differences between $T_{\mathrm{C}}$ and $T_{\mathrm{M}-\mathrm{I}}[2-4]$. The FM metallic state at low temperatures was explained in terms of the double-exchange (DE) interaction arising from strong on-site Hund's rule coupling between $\mathrm{Mn}^{3+}$ and $\mathrm{Mn}^{4+}$ ions [5]. Hopping of the $e_{\mathrm{g}}$ electron of $\mathrm{Mn}^{3+}$ along $\mathrm{Mn}-\mathrm{O}-\mathrm{Mn}$ bonds gives rise to metallic conductivity in the ferromagnetic region. Although the DE model provides a qualitatively correct description of these materials, more complex models incorporating the dynamic Jahn-Teller effect [6] and phase separation have been proposed [7]. Above $T_{\mathrm{C}}$ the electrical conduction in $\mathrm{La}_{0.7} \mathrm{Ca}_{0.3} \mathrm{MnO}_{3}$ is shown to follow the thermally activated hopping conduction law [8] $\rho=\rho_{0} \exp (E / k T)$, with the activation energy $E \approx 0.1 \mathrm{eV}$, as well as Mott's variable-range-hopping (VRH) conduction rule [9], $\rho=\rho_{\infty} \exp \left(T_{0} / T\right)^{1 / 4}$, where $T_{0}$ is the Mott characteristic temperature. Although it has been well recognized that polarons exist in the PM phase of the manganites, the true nature of this polaronic phase and the transport process is still a matter of discussion [10].

Only a few studies have been carried out on Sn-doped (La,Ca) $\mathrm{MnO}_{3}$ compounds [11-15]. They are contradictory with respect to the Sn solubility limit but one can infer that this limit is in the range of only few at.\% of Sn. It is now generally accepted that Sn substitutes for $\mathrm{Mn}^{4+}$ [12], forming $\mathrm{Mn}^{3+} / \mathrm{Sn}^{4+}$ pairs which do not support DE interaction. In addition, due to their larger size, $\mathrm{Sn}^{4+}$ cations introduce local lattice distortions. The main point of our studies was to prepare single phase polycrystalline $\left(\mathrm{La}_{0.67} \mathrm{Ca}_{0.33}\right)\left(\mathrm{Mn}_{1-x} \mathrm{Sn}_{x}\right) \mathrm{O}_{3}$ compounds and to carefully characterize their structural, magnetic, and electrical transport properties.

\section{Experimental}

Polycrystalline samples of $\left(\mathrm{La}_{0.67} \mathrm{Ca}_{0.33}\right)\left(\mathrm{Mn}_{1-x} \mathrm{Sn}_{x}\right) \mathrm{O}_{3-\delta}(x=0,0.01$, and $0.03)$ were prepared by a sol-gel method. A calculated amount of metallic Sn was added to $5 \mathrm{ml}$ of concentrated nitric acid and a colloidal suspension of $\mathrm{SnO}_{2}$ was obtained. Acetic acid was added to bring the total volume to $100 \mathrm{ml}$. The solution was heated and stoichiometric amounts of $\mathrm{La}_{2} \mathrm{O}_{3}, \mathrm{CaCO}_{3}$, and $\mathrm{MnCO}_{3}$ were 
given into the solution under stirring. $45 \mathrm{~g}$ of citric acid and $11 \mathrm{~g}$ ethylene glycol were added into the mixture and the solution was slowly heated until a brown gel was formed. The gel was dried at $130^{\circ} \mathrm{C}$ for $90 \mathrm{~min}$, then slowly heated to $270^{\circ} \mathrm{C}$ (heating rate $10 \mathrm{~K} \mathrm{~h}^{-1}$ ) and kept at this temperature for one hour. The temperature was then increased to $730^{\circ} \mathrm{C}$ within 10 hours, kept at this temperature for 12 hours and cooled to room temperature within another 12 hours. The calcined samples were milled, pressed into pellets at $0.4 \mathrm{GPa}$ and sintered in air at $1450^{\circ} \mathrm{C}$ for 50 hours. The heating and the cooling rates were $3 \mathrm{~K} \mathrm{~min}^{-1}$. The higher than usually sintering temperature was found necessary to completely dissolve up to 3 at.\% of $\mathrm{Sn}$ in $\mathrm{La}_{0.67} \mathrm{Ca}_{0.33} \mathrm{MnO}_{3}$ compound. For the two border compounds in the series with $x=0$ and $x=0.03$ the oxygen off-stoichiometry parameter $\delta$ was determined by a iodometric titration method and found to be 0.06(1) and 0.0055(10), respectively. The same $\delta$ value was assumed for the $x=0.01$ compound.

The quality of the samples was checked by X-ray powder diffraction. The X-ray diffraction (XRD) patterns were collected with a D5000 Siemens diffractometer (using $\mathrm{Cu} K_{\alpha}$ radiation and a graphite secondary monochromator) from $12^{\circ}$ to $120^{\circ}$ in steps of $0.02^{\circ}$ in $2 \theta$ and $12 \mathrm{~s}$ per step. The refinement of the XRD patterns was carried out by the Fullprof program [16] assuming the perovskite $\mathrm{GdFeO}_{3}$ - orthorhombic type structure and using the Pbnm space group.

The microstructure was studied by scanning electron microscopy (JSM-6400 JEOL, Japan).

Magnetic characterization of the samples was performed on a conventional AC susceptometer with an excitation field of 0.5 Oe and a frequency of $188.9 \mathrm{~Hz}$ as well as on a DC VSM magnetometer. The DC magnetization was recorded during heating in applied DC fields of 1000 Oe after zero-field cooling (ZFC) of the sample down to $78 \mathrm{~K}$. The temperature dependence of the resistance $R$ was measured by the standard four probe AC method at zero external field during cooling the sample.

Specific heat measurements were carried out by the standard semi-adiabatic heat pulse method in the temperature range from 80 to $300 \mathrm{~K}$ during warming the sample.

\section{Results and analysis}

\subsection{Structure}

The XRD patterns of all $\left(\mathrm{La}_{0.67} \mathrm{Ca}_{0.33}\right)\left(\mathrm{Mn}_{1-x} \mathrm{Sn}_{x}\right) \mathrm{O}_{3-\delta}$ compounds annealed in air at $1450^{\circ} \mathrm{C}$ for 50 hours showed that all of them were practically single phase. The volume fraction calculated by the Rietveld method of the only detected but hardly visible $\mathrm{CaO}$ type impurity phase was below $1 \%$. As an example, a part of XRD pattern of the $x=0.03$ compound is seen in Fig. 1. The vertical arrow indicates the largest peak of the $\mathrm{CaO}$ impurity phase with the refined volume fraction of $0.4(2) \%$. One could take into account this lost of calcium 


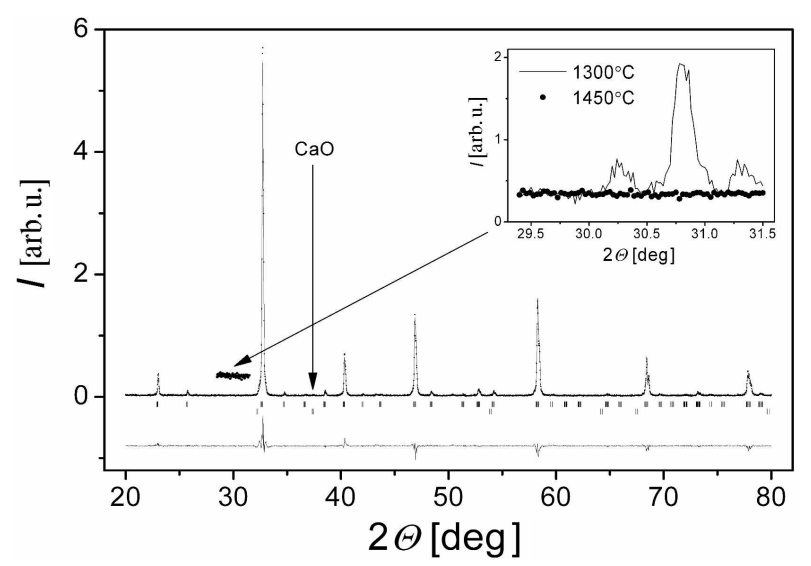

Fig. 1. Part of an XRD pattern of the $\left(\mathrm{La}_{0.67} \mathrm{Ca}_{0.323}\right)\left(\mathrm{Mn}_{0.97} \mathrm{Sn}_{0.03}\right) \mathrm{O}_{2.94}$ compound at room temperature. The arrow indicates the largest peak of the $\mathrm{CaO}$ impurity phase. The inset shows an enlarged part of the pattern measured with much better statistics and with superimposed peaks of an unwanted tin-rich impurity phase (continuous line).

from the manganite phase and calculate the corrected calcium stoichiometry as equal to $0.323(4)$ instead of supposed value 0.33 . Our preliminary attempts to introduce 3-6 at.\% of Sn into $\left(\mathrm{La}_{0.67} \mathrm{Ca}_{0.33}\right) \mathrm{MnO}_{3}$ compound at lower sintering temperature $\left(1300^{\circ} \mathrm{C}\right)$ showed that part of the tin precipitates in an unidentified impurity phase with the most intense peak at about 30.8 degree (as shown in the inset of Fig. 1). To exclude the existence of this impurity phase the 29-31.5 degree range was re-measured with much longer accumulation time per step (210 s), but no additional peaks were revealed. This additional points denoted by larger solid circles are also seen in Fig. 1. The inset of Fig. 1 shows again these additional points but superimposed on the scaled up part of the XRD pattern of another $x=0.03$ compound (denoted by continuous line) annealed in air at $1300^{\circ} \mathrm{C}$ for 10 hours.

No traces of the tin-rich impurity phase were found for the $x=0.01$ compound [17]. Mössbauer spectroscopy studies on ${ }^{119} \mathrm{Sn}$ isotope have clearly confirmed that 1 at.\% of tin has been completely dissolved in $\mathrm{La}_{0.67} \mathrm{Ca}_{0.33} \mathrm{MnO}_{3}$ compound. For the $x=0.01$ and $x=0$ compounds the same volume fractions of the $\mathrm{CaO}$ impurity phase of $0.9(2) \%$ were found. The corrected calcium stoichiometry for these compounds should be 0.314(4). Refined lattice parameters and unit cell volumes are given in Table for all three compounds.

To our knowledge there is only little structural information available in literature for oxygen deficient $\left(\mathrm{La}_{0.67} \mathrm{Ca}_{0.33}\right) \mathrm{MnO}_{3-\delta}$, but there are many data for the compounds with exact or "excess" oxygen content. Relating the unit cell volume of the $\left(\mathrm{La}_{0.66} \mathrm{Ca}_{0.314}\right) \mathrm{MnO}_{2.94}$ compound from Table to the unit cell volume of the $\left(\mathrm{La}_{0.66} \mathrm{Ca}_{0.34}\right) \mathrm{MnO}_{3}$ compound with exact oxygen stoichiometry $\left(V=230.132 \AA^{3}\right)$ [18] shows that the stoichiometric compound has an about $0.9 \%$ smaller volume. 


\section{TABLE}

The unit cell parameters $a, b, c$ and volume $V$ for $\left(\mathrm{La}_{0.67} \mathrm{Ca}_{0.33}\right)\left(\mathrm{Mn}_{1-x} \mathrm{Sn}_{x}\right) \mathrm{O}_{3-\delta}$ compounds with $x=0,0.01$ and 0.03 , annealed in air at $1450^{\circ} \mathrm{C}$ for 50 hours.

\begin{tabular}{l|c|c|c}
\hline \hline \multicolumn{1}{c|}{$x$} & 0.0 & 0.01 & 0.03 \\
\hline$a[\AA]$ & $5.4862(1)$ & $5.4910(3)$ & $5.4940(3)$ \\
$b[\AA]$ & $5.4713(1)$ & $5.4791(3)$ & $5.4869(3)$ \\
$c[\AA]$ & $7.7346(1)$ & $7.7452(4)$ & $7.7537(4)$ \\
$V\left[\AA^{3}\right]$ & $232.17(1)$ & $233.02(2)$ & $233.73(2)$
\end{tabular}

For oxygen over-stoichiometric compounds with $\delta<0$, the unit cell volume decreases with increasing $|\delta|[18]$, confirming that the additional holes are doped uniformly by cation-vacancy defects and that the chemical formula for the compound should rather read $\left(\mathrm{La}_{0.67} \mathrm{Ca}_{0.33}\right)_{3 /(3-\delta)} \mathrm{Mn}_{3 /(3-\delta)} \mathrm{O}_{3}$. The clearly larger unit cell volumes of the oxygen under-stoichiometric compounds (Table) reveal that vacancies on oxygen sites are formed and that the formulas for these compounds should read $\left(\mathrm{La}_{0.67} \mathrm{Ca}_{0.33}\right)\left(\mathrm{Mn}_{1-x} \mathrm{Sn}_{x}\right) \mathrm{O}_{3-\delta}$. The XRD patterns of all of the $\left(\mathrm{La}_{0.67} \mathrm{Ca}_{0.33}\right)\left(\mathrm{Mn}_{1-x} \mathrm{Sn}_{x}\right) \mathrm{O}_{3-\delta}$ compounds do not show noticeable line broadening effects above the instrumental width. Thus no significant microstrains in grains exist. Minimum grains diameter of about $2 \mu \mathrm{m}$ was estimated.
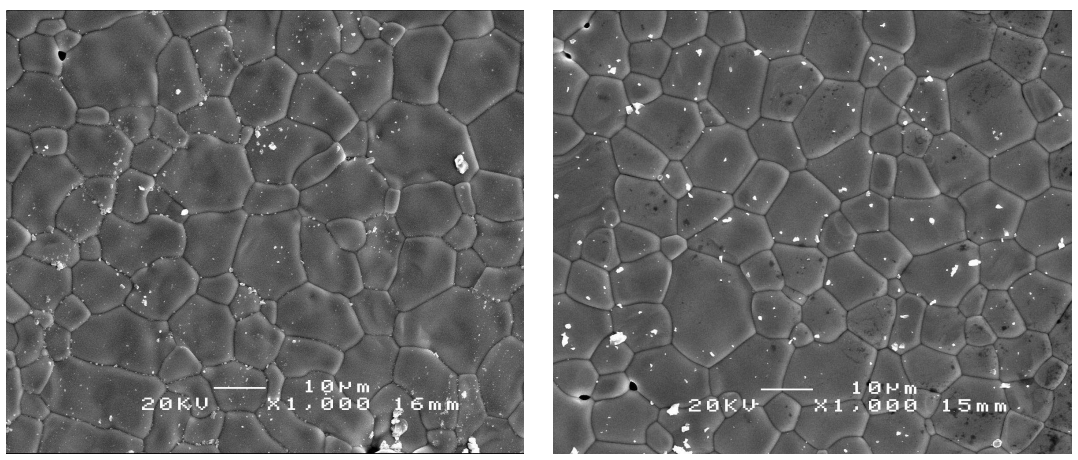

Fig. 2. Scanning electron micrographs of $\left(\mathrm{La}_{0.67} \mathrm{Ca}_{0.314}\right) \mathrm{MnO}_{2.94}$ (left part) and of $\left(\mathrm{La}_{0.67} \mathrm{Ca}_{0.323}\right)\left(\mathrm{Mn}_{0.97} \mathrm{Sn}_{0.03}\right) \mathrm{O}_{2.94}$ (right part) compounds.

The scanning electron microscopy (SEM) images shown in Fig. 2 correspond to compounds with $x=0$ (left) and $x=0.03$ (right), respectively. In both compounds the average grain diameter is about $11 \mu \mathrm{m}$ but the grain size varies widely. The shape of the grains is quite similar in both compounds since the compounds were sintered under the same conditions. It is also natural to expect that the SEM image for the $x=0.01$ compound, prepared under the same conditions as the other two compounds, would be similar. 


\subsection{Magnetic and transport properties}

Figure 3 shows the temperature dependence of the AC magnetic susceptibility $\left(\chi_{\mathrm{AC}}^{\prime}\right)$ for compounds with $x=0,0.01$, and 0.03. A non-systematic influence of Sn doping on the $\chi_{\mathrm{AC}}^{\prime}$ curves may be observed. The maximum of $\chi_{\mathrm{AC}}^{\prime}(T)$ first increases in amplitude for $x=0.01$ and then decreases for $x=0.03$. The parent $x=0$ compound exhibits a sharp ferromagnetic-paramagnetic transition. With increasing Sn substitution for Mn the magnetic transition becomes broader and the transition temperature decreases. Defining the critical temperature $T_{\mathrm{C}}^{\chi}$ as the inflection point of the $\chi_{\mathrm{AC}}^{\prime}(T)$ curve the following critical temperatures, $219.7 \mathrm{~K}$, 176.4 K, and 142.6 K were obtained for the $x=0, x=0.01$, and $x=0.03$ compounds, respectively. The $T_{\mathrm{C}}^{\chi}$ values clearly show a nonlinear decrease with $x$.

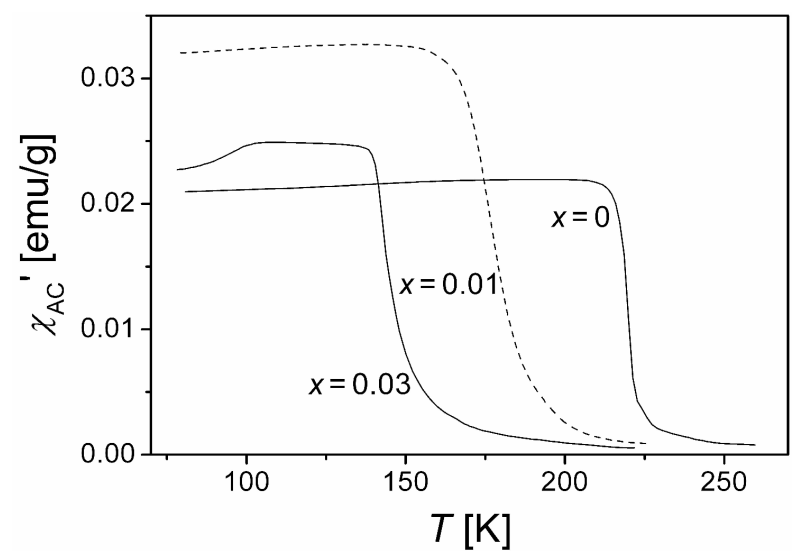

Fig. 3. Temperature dependence of $\chi_{\mathrm{AC}}^{\prime}$ for $\left(\mathrm{La}_{0.67} \mathrm{Ca}_{0.33}\right)\left(\mathrm{Mn}_{1-x} \mathrm{Sn}_{x}\right) \mathrm{O}_{3-\delta}$ with $x=0,0.01$, and 0.03 .

Quite similar results for $T_{\mathrm{C}}$ 's were obtained from magnetization $(M)$ measurements performed on a VSM magnetometer in an applied field of 1000 Oe using a ZFC procedure. The superimposed magnetization and resistance versus $T$ for all $\left(\mathrm{La}_{0.67} \mathrm{Ca}_{0.33}\right)\left(\mathrm{Mn}_{1-x} \mathrm{Sn}_{x}\right) \mathrm{O}_{3-\delta}$ compounds are shown for comparison in Fig. 4. The typically defined $T_{\mathrm{C}}$ values (as the temperature corresponding to the minimum of $\mathrm{d} M / \mathrm{d} T$ ) for the $x=0, x=0.01$, and $x=0.03$ compounds, are equal to $223.2 \mathrm{~K}, 172.3 \mathrm{~K}$, and $135 \mathrm{~K}$. It is interesting to note that the drop of $50.9 \mathrm{~K}$ in the $T_{\mathrm{C}}$ value between $x=0$ and $x=0.01$ is larger than between the $x=0.01$ and $x=0.03$ compounds $(37.3 \mathrm{~K})$. To estimate quantitatively the spreads of magnetic transitions $\left(\Delta T_{\mathrm{C}}\right)$ it is useful to define also the "upper" $T_{\mathrm{C}}^{\mathrm{up}}$ as the intersection of the straight line fitted to magnetization points with the horizontal $M=0$ line (as the extrapolation of the maximal slope of the magnetization curve to the $T$ axes). Next, defining $\Delta T_{\mathrm{C}}$ as the difference $T_{\mathrm{c}}^{\mathrm{up}}-T_{\mathrm{C}}$, one finds that it equals $2.5 \mathrm{~K}$, $20 \mathrm{~K}$, and $25 \mathrm{~K}$ for the $x=0, x=0.01$, and $x=0.03$ compounds, respectively. Also the $\Delta T_{\mathrm{C}}$ values show a strongly nonlinear increase with $x$. 


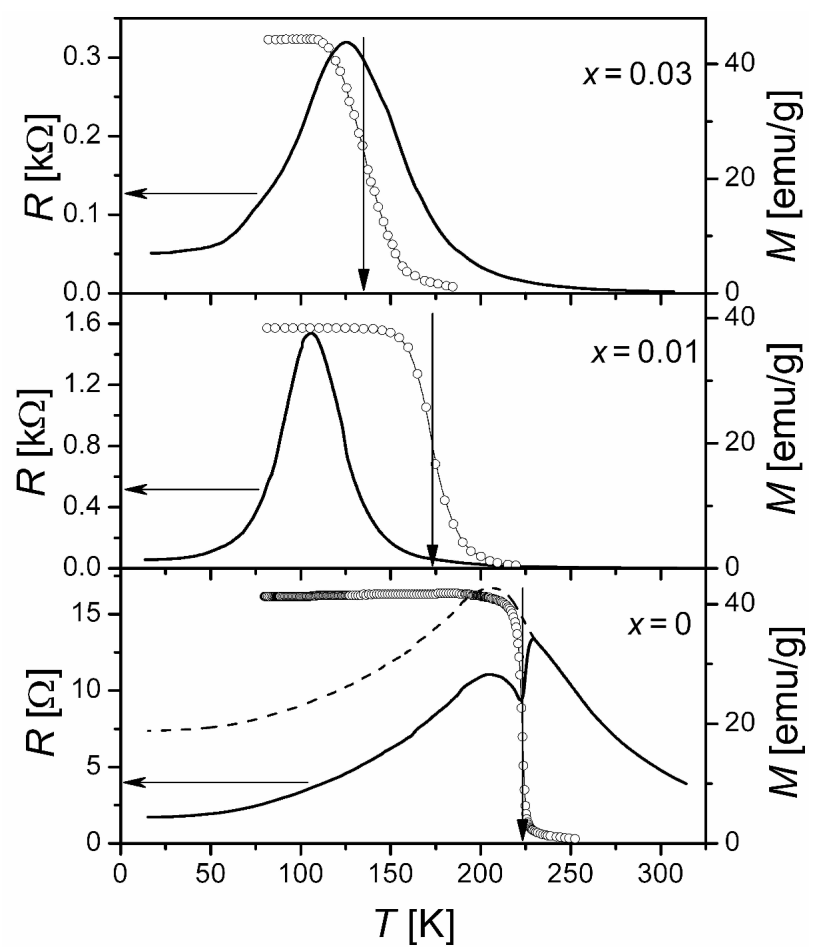

Fig. 4. Temperature dependence of $M$ and $R$ curves for $\left(\mathrm{La}_{0.67} \mathrm{Ca}_{0.33}\right)\left(\mathrm{Mn}_{1-x} \mathrm{Sn}_{x}\right) \mathrm{O}_{3-\delta}$ with $x=0,0.01$, and 0.03 . Vertical arrows indicate $T_{\mathrm{C}}$ values. The dashed line denotes $R_{\mathrm{C}}(T)$ curve corrected for the magnetic effects observed at $T_{\mathrm{C}}$.

The $R(T)$ curves demonstrate even more interesting changes with $x$. For the $x=0.03$ compound the resistance curve shows a transition from a metallic-like to an insulating state $(\mathrm{M}-\mathrm{I})$ at the temperature $T_{\mathrm{M}-\mathrm{I}}$ of about $125 \mathrm{~K}$ (defined as the maximum of the $R(T)$ ). The maximum value of the resistance $R_{\max }$ is $320 \Omega$. Moreover, the resistance at the lowest investigated temperature $(17 \mathrm{~K})$ is 22 times higher than the one at room temperature. $T_{\mathrm{M}-\mathrm{I}}$ is $10 \mathrm{~K}$ lower than $T_{\mathrm{C}}$ for this compound. For the $x=0.01$ compound the $R(T)$ curve shows a sharper $\mathrm{M}-\mathrm{I}$ transition at clearly lower $T_{\mathrm{M}-\mathrm{I}}$ of about $106 \mathrm{~K}$ than for $x=0.03$ compound. Surprisingly the $R_{\max }$ has larger value than for $x=0.03$ compound of $1540 \Omega$. Also the resistance at the lowest investigated temperature $(14 \mathrm{~K})$ is larger than for $x=0.03$ compound and is 29 times higher than the one at room temperature. $T_{\mathrm{M}-\mathrm{I}}$ is $66.3 \mathrm{~K}$ lower than $T_{\mathrm{C}}$ for this compound. For the $x=0$ compound the situation is more complicated. The $R(T)$ curve shows formally two M-I transitions (defined as the maxima of the $R(T)$ ). One sharp transition occurs at $229 \mathrm{~K}$, and a second, broader one at about $205 \mathrm{~K}$. Below the upper $T_{\mathrm{M}-\mathrm{I}}$ the resistance falls drastically. This coincides with the sharp increase in the magnetization and indicates that the sharp drop in resistance below this temperature is associated 
with ferromagnetic ordering. As a consequence, the smaller and broader peak at lower temperature should be, by analogy with other two compounds, identified as the actual M-I transition temperature. The $R_{\max }$ at this temperature equals to $11 \Omega$. Only in this case the resistance at the lowest investigated temperature $(15 \mathrm{~K})$ is smaller (about 3 times) than the one at room temperature. $T_{\mathrm{M}-\mathrm{I}}$ is $18.2 \mathrm{~K}$ lower than $T_{\mathrm{C}}$ for this compound.

Since the hopping mechanism of conductivity plays a dominant role in the insulating state of the manganites, the experimental resistance data were plotted as $\ln R$ versus $T^{-1 / 4}$ in order to examine the applicability of the Mott VRH model $R_{\mathrm{VRH}}=R_{\infty} \exp \left(T_{0} / T\right)^{1 / 4}[9]$. It was found that starting from the vicinity of the corresponding $T_{\mathrm{C}}$ up to above room temperature the experimental points follow straight lines and obey the Mott law very well. In these temperature ranges the resistance data were fitted by straight lines using linear regression analysis. The values of $R_{\infty}$ and $T_{0}$ parameters were derived from the regression analysis.

In order to reveal the effect of magnetic ordering on the $R(T)$ dependence the difference curves $\Delta R(T)=R_{\infty} \exp \left(T_{0} / T\right)^{1 / 4}-R(T)$ were calculated for all compounds and plotted in Fig. 5. Such plots show the range of applicability of the VRH model very well. In the case of the $x=0.01$ compound this range is largest and extends practically to $T_{\mathrm{C}}$. In case of the $x=0$ and $x=0.03$

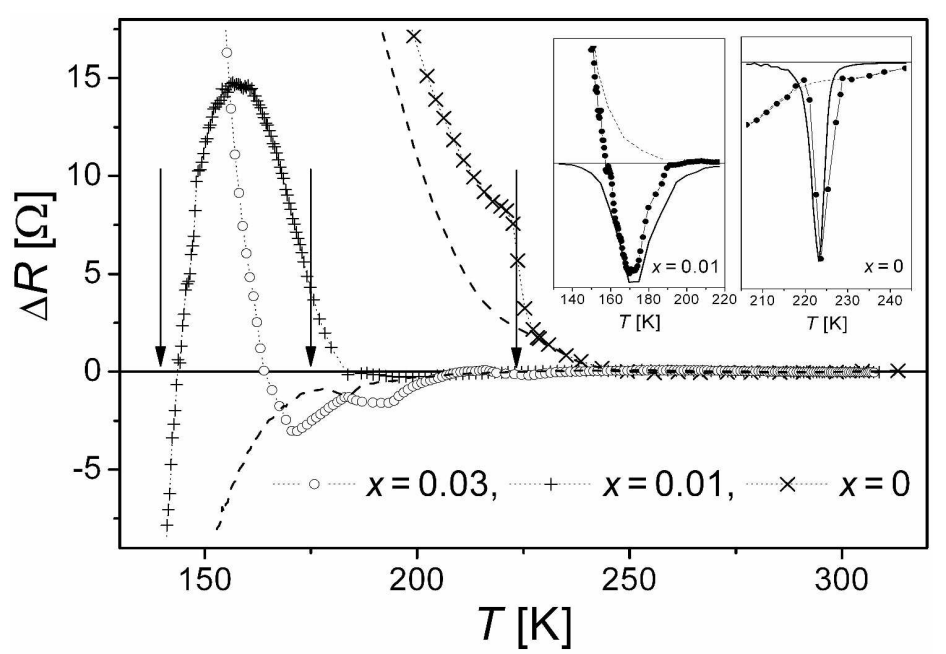

Fig. 5. Temperature dependence of $\Delta R$ for $\left(\mathrm{La}_{0.67} \mathrm{Ca}_{0.33}\right)\left(\mathrm{Mn}_{1-x} \mathrm{Sn}_{x}\right) \mathrm{O}_{3-\delta}$ with $x=0,0.01$, and 0.03. Dashed curves show the effect of subtraction of the calculated magnetic contributions from $\Delta R(T)$ curves. Vertical arrows indicate $T_{\mathrm{C}}$ values. The insets show the $\mathrm{d}(\Delta R) / \mathrm{d} T$ (denoted by filled circles) with superimposed $\mathrm{d} M / \mathrm{d} T$ curves (solid lines) for $x=0.01$ (left inset) and $x=0$ (right). Subtracted base line curves for $\mathrm{d}(\Delta R) / \mathrm{d} T$ are denoted by dashed lines. 
compounds deviations with opposite signs appear at larger distances from the corresponding $T_{\mathrm{C}}$. These plots also reveal that for all compounds the resistance falls drastically just above $T_{\mathrm{C}}$. This coincides with the sharp increase in the magnetization (Fig. 4). The close similarity of the $\mathrm{d}(\Delta R) / \mathrm{d} T$ and $\mathrm{d} M / \mathrm{d} T$ peaks for the $x=0$ and $x=0.01$ compounds shown in the insets (Fig. 5) indicates that the peak in $\mathrm{d}(\Delta R) / \mathrm{d} T$ at $T_{\mathrm{C}}$ originates solely from the spontaneous magnetization change $\mathrm{d} M_{\mathrm{S}} / \mathrm{d} T$. By subtracting the sloping base lines from the $\mathrm{d}(\Delta R) / \mathrm{d} T$ curves the peaks resulting exclusively from the $M_{\mathrm{S}}$ versus $T$ change can be separated. Denoting this function by $\mathrm{d} R_{\mathrm{S}} / \mathrm{d} T$ (as due to $\mathrm{d} M_{\mathrm{S}} / \mathrm{d} T$ ) one obtains by numerical integration the contribution to the resistance, which is due to the spontaneous magnetization $R_{\mathrm{S}}(T)=-\int_{+\infty}^{T}\left(\mathrm{~d} R_{\mathrm{S}} / \mathrm{d} T\right) \mathrm{d} T$. Such procedure is only possible for compounds with well separated $T_{\mathrm{C}}$ and $T_{\mathrm{M}-\mathrm{I}}$ temperatures. The curves denoted by dashed lines in Fig. 5 were obtained by calculating $R_{\mathrm{S}}(T)$ values for the $x=0$ and $x=0.01$ compounds and adding to the corresponding $\Delta R(T)$ functions. The differences between the $\Delta R(T)$ function and the calculated dashed curves shows the effect of magnetic ordering on the resistance. The magnitude of the magnetic contribution to the resistance is clearly larger for the $x=0.01$ compound $\left(\left|R_{\mathrm{S}}(0)\right|=22.4 \Omega\right)$ than for $x=0$ one $\left(\left|R_{\mathrm{S}}(0)\right|=5.7 \Omega\right)$. This effect was clearly visible in Fig. 3 for the $x=0$ compound but even though larger in magnitude was completely unnoticeable for $x=0.01$ one. By subtracting the calculated $R_{\mathrm{S}}(T)$ contribution from the corresponding experimental $R(T)$ values one may obtain the corrected $R_{\mathrm{C}}(T)$ curve for the hypothetical compound which do not show the colossal magnetoresistance effect at $T_{\mathrm{C}}$. The $R_{\mathrm{C}}(T)$ values for $x=0$ compound are denoted in Fig. 3 by the dashed curve. This curve shows only one of the two M-I transitions, namely the one at about $205 \mathrm{~K}$. The $R_{\mathrm{C}}(T)$ curve for the $x=0.01$ compound was not plotted because it was practically undistinguishable from the measured $R(T)$ curve.

\subsection{Specific heat}

Figure 6 shows the temperature dependence of the specific heat of $\left(\mathrm{La}_{0.67} \mathrm{Ca}_{0.33}\right)\left(\mathrm{Mn}_{1-x} \mathrm{Sn}_{x}\right) \mathrm{O}_{3-\delta}$. For the Sn-free sample, the $c_{p}(T)$ curve exhibits a sharp peak at $T_{\mathrm{C}}$. With increasing Sn content the peaks broaden and for $x=0.03$ the peak is so broad that it is hardly detectable. In order to separate the lattice contributions and to estimate the magnetic contributions to the specific heat $\left(c_{\mathrm{m}}\right)$ for the given compound the smoothed $c_{p}(T)$ curve with the most separated $c_{p}(T)$ peak was subtracted and then the background of the $c_{\mathrm{m}}$ peak was corrected by straight line subtraction. For example from the $c_{p}(T)$ curve for $x=0$ the smoothed curve for $x=0.03$ was subtracted.

The peak temperature, $T_{\mathrm{p}}$ of the $c_{\mathrm{m}}(T)$ curve for $x=0$ coincides perfectly with the $T_{\mathrm{C}}$ derived from magnetization measurements. This implies that the peak is associated with the magnetic transition. In case of the $x=0.01$ compound, however, some mismatch between corresponding $T_{\mathrm{p}}$ and $T_{\mathrm{C}}$ temperatures 


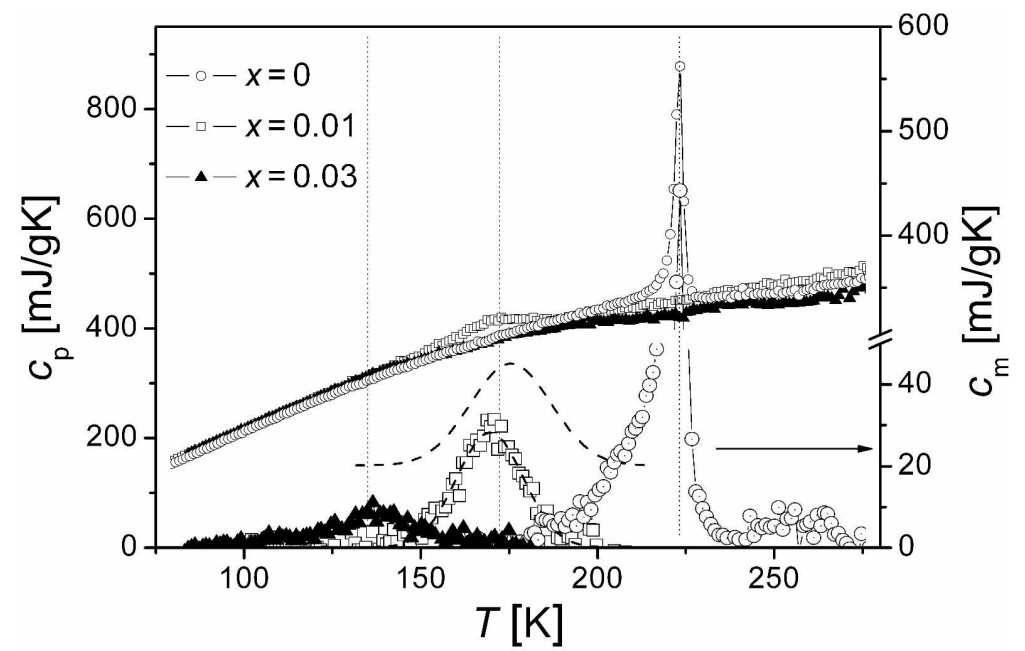

Fig. 6. Temperature dependence of specific heat of $\left(\mathrm{La}_{0.67} \mathrm{Ca}_{0.33}\right)\left(\mathrm{Mn}_{1-x} \mathrm{Sn}_{x}\right) \mathrm{O}_{3-\delta}$ with $x=0,0.01$, and 0.03 . Vertical dotted lines indicate $T_{\mathrm{C}}$ values (from magnetization measurements). Separated and enlarged peaks show the magnetic contributions $\left(c_{\mathrm{m}}\right)$ to the specific heat. Solid lines are to guide the eyes. The meaning of dashed lines is explained in text.

is seen. In order to understand the origin of this mismatch one should realize that in the simple model $c_{\mathrm{m}}(T)$ is proportional to $\mathrm{d}\left(M_{\mathrm{S}}{ }^{2}(T)\right) / \mathrm{d} T$ and not to the $\mathrm{d}(M(T)) / \mathrm{d} T$ function from which the $T_{\mathrm{C}}$ value was derived. For the estimation of the importance of this effect the Gaussian peak shape function was fitted to the experimental points for the $x=0.01$ sample and plotted in Fig. 6 (dashed line). A $T_{\mathrm{p}}$ of $169.6(3) \mathrm{K}$ and a full width at half maximum (FWHM) value of 22.2(8) K were determined from these calculations. Next, by integration of the Gaussian function and by taking the square root, $M_{\mathrm{S}}(T)$ was found from which the $-\mathrm{d}\left(M_{\mathrm{S}}(T)\right) / \mathrm{d} T$ shape function was calculated and plotted with some vertical offset (dashed line) in Fig. 6. It is even too far shifted in the right direction (new $T_{\mathrm{p}}$ of $175.5 \mathrm{~K}$ ) as compared with the $T_{\mathrm{C}}=172.3 \mathrm{~K}$. In summary, taking into account FWHM of the $c_{\mathrm{m}}(T)$ peak for the $x=0.01$ compound and experimental errors even larger $T_{\mathrm{C}}-T_{\mathrm{p}}(=2.7 \mathrm{~K})$ is expected.

This rough analysis allows the conclusion that the $T_{\mathrm{C}}-T_{\mathrm{p}}$ difference always exists and should be taken into account in determination of $T_{\mathrm{C}}$ from the specific heat measurements. Generally, this difference should scale with the value of the FWHM for the given $c_{\mathrm{m}}$ peak. One should note that no anomalies in $c_{p}(T)$ curves are seen for the $x=0.01$ and $x=0$ compounds at $T_{\mathrm{M}-\mathrm{I}}$. In the case of $x=0.03$ agreement of $T_{\mathrm{p}}$ and $T_{\mathrm{C}}$ temperatures can be assumed even though it is hard to determine the precise value of $T_{\mathrm{p}}$ in this case. When the part of $\mathrm{Mn}$ is substituted by $\mathrm{Sn}$, the $c_{\mathrm{m}}$ peak broadens and the latent heat for the phase transition decreases 
as revealed by the decreasing area under the peak with increasing $x$. The estimated ratio of the latent heats for the $x=0,0.01$, and 0.03 compounds is approximately $8: 3.5: 1$.

\section{Discussion}

Since the oxygen stoichiometry, which is a function of the synthesis condition, strongly affects the properties of manganites, all three compounds studied were prepared under strictly the same procedure. Therefore the properties of the compounds vary only due to the different substitution level Sn for Mn. The structural characterization confirmed that tin was completely dissolved in the structure and good quality compounds with large grains (diameter $11 \mu \mathrm{m}$ ) were formed.

A high sintering temperature $\left(1450^{\circ} \mathrm{C}\right)$ is needed to substitute $\mathrm{Sn}$ for Mn but this results in an oxygen deficiency $\delta(0.06)$. The vacancies connected with positive $\delta$ values formed on the oxygen sites introduce local structural distortions and electronic inhomogeneities, which could affect structural, transport, and magnetic properties. What is also very important $\delta$ decreases the effective hole doping $(h)$ by amount of $2 \delta$. Equivalent hole doping would be obtained for stoichiometric compound with a Ca content equal to 0.21 . This value shifts our compounds close to the border line of the stability of the FM metallic state, which locates for the stoichiometric compounds slightly below 0.2 on the phase diagram [19] and separates the FM metallic from the FM insulating states. A low value of hole doping explains the low value of $T_{\mathrm{C}}(223.2 \mathrm{~K})$ determined for the $\left(\mathrm{La}_{0.66} \mathrm{Ca}_{0.314}\right) \mathrm{MnO}_{2.94}$ compound. It is only slightly larger than expected for the $h=0.21$ from the phase diagram. The proximity to the FM metal-FM insulator border explains the large sensitivity of the compounds to relatively low levels of Sn doping and the appearance of the FM insulating state in between the $T_{\mathrm{M}-\mathrm{I}}$ and $T_{\mathrm{C}}$ temperatures.

The introduction of diamagnetic $\mathrm{Sn}$ causes an exclusive replacement of $\mathrm{Mn}^{4+}$ by $\mathrm{Sn}^{4+}$ cations. This leads to an increase in the $\mathrm{Mn}^{3+} / \mathrm{Mn}^{4+}$ ratio and reduces the number of available hopping sites for electrons. The $\mathrm{Sn}^{4+}$ ions do not participate in conduction and cannot be coupled magnetically to $\mathrm{Mn}^{3+} / \mathrm{Mn}^{4+}$ ions causing partial suppression of the conductivity and the ferromagnetic double exchange. In addition, due to their larger size, $\mathrm{Sn}^{4+}$ cations introduce microscopic strains and local lattice distortions which slow down the electron hopping, and as a result, lead to a strong suppression of $T_{\mathrm{C}}$ and $T_{\mathrm{M}-\mathrm{I}}$.

All investigated $\left(\mathrm{La}_{0.67} \mathrm{Ca}_{0.33}\right)\left(\mathrm{Mn}_{1-x} \mathrm{Sn}_{x}\right) \mathrm{O}_{3-\delta}$ compounds are paramagnetic insulators at elevated temperatures and conduct via thermally assisted variable-range-hopping [9]. Assuming that above $T_{\mathrm{C}}$, the $e_{\mathrm{g}}$ electrons are localized by random spin-dependent potential, Viret et al. [20] have obtained an expression for the $T_{0}$ parameter in the Mott equation $T_{0}=171 U_{\mathrm{m}} v / \xi^{3} k_{\mathrm{B}}$, where $U_{\mathrm{m}} \approx 2 \mathrm{eV}$ is the amplitude of the magnetic potential, $v$ is the unit cell volume per manganese ion, $\xi$ is the localization length and $k_{\mathrm{B}}$ is the Boltzmann constant. Using the calculated $T_{0}$ values, obtained from the fit to the VRH model, localization lengths $\xi$ 
equal to $2.2 \AA, 1.33 \AA$, and $1.26 \AA$ were estimated for compounds with $x=0,0.01$, and 0.03 , respectively. Using the same method Viret et al. [20] obtained $\xi=4.5 \AA$ for $\mathrm{La}_{0.67} \mathrm{Sr}_{0.33} \mathrm{MnO}_{3}$ and $\xi=0.8 \AA$ for $\mathrm{Pr}_{0.67} \mathrm{~Pb}_{0.33} \mathrm{MnO}_{3}$. One should note that with increasing Sn content the parameter $\xi$ decreases indicating the progressive localization of the charge carriers in the paramagnetic state. There is a large decrease in the localization lengths between the $x=0$ and $x=0.01$ compounds and only a moderate one between the $x=0.01$ and $x=0.03$ compounds. This corresponds well with the observed changes in resistance.

The $x=0$ and $x=0.01$ compounds exhibited anomalies of $R(T)$ in accordance with the onset of the spontaneous magnetization $M_{\mathrm{S}}$ at corresponding $T_{\mathrm{C}}$ and offered the unique possibility to separate the contributions to $R_{\mathrm{S}}(T)$ and to estimate the magnitude of this effect. The separation of the $R_{\mathrm{S}}(T)$ contribution was much more precise in the case of the $x=0$ compound in which the magnetic transition at $T_{\mathrm{C}}$ was very sharp and $T_{\mathrm{C}}$ and $T_{\mathrm{M}-\mathrm{I}}$ temperatures were still reasonably well separated $(\approx 20 \mathrm{~K})$. This was also due to comparable magnitudes of the measured $R(T)$ and calculated $R_{\mathrm{S}}(T)$ contributions. For the quantitative description of the magnitude of this effect it is useful to define the parameter $\eta(T)=100 \frac{\left|R_{\mathrm{S}}(T)\right|}{R_{\mathrm{C}}(T)}$, which has the meaning of $100 \frac{\left[R(0, T)-R\left(M_{\mathrm{S}}, T\right)\right]}{R(0, T)}$ ratio, where $R\left(M_{\mathrm{S}}, T\right)$ is the measured resistance $(R(T))$ but $\left.R(0, T) \stackrel{(=}{=} R_{\mathrm{C}}(T)=R\left(M_{\mathrm{S}}, T\right)-\left|R_{\mathrm{S}}(T)\right|\right)$ is the one corrected for $M_{\mathrm{S}}$ effects. In analogy to the normal magnetoresistance definition it may be called the "intrinsic" or spontaneous magnetoresistance parameter. The $\eta(T)$ and $-R_{\mathrm{S}}(T)$ dependences for the $x=0$ and $x=0.01$ compounds are plotted in Fig. 7. In both cases comparable "colossal magnetoresistance" effects are observed at $T_{\mathrm{C}}$ temperatures.

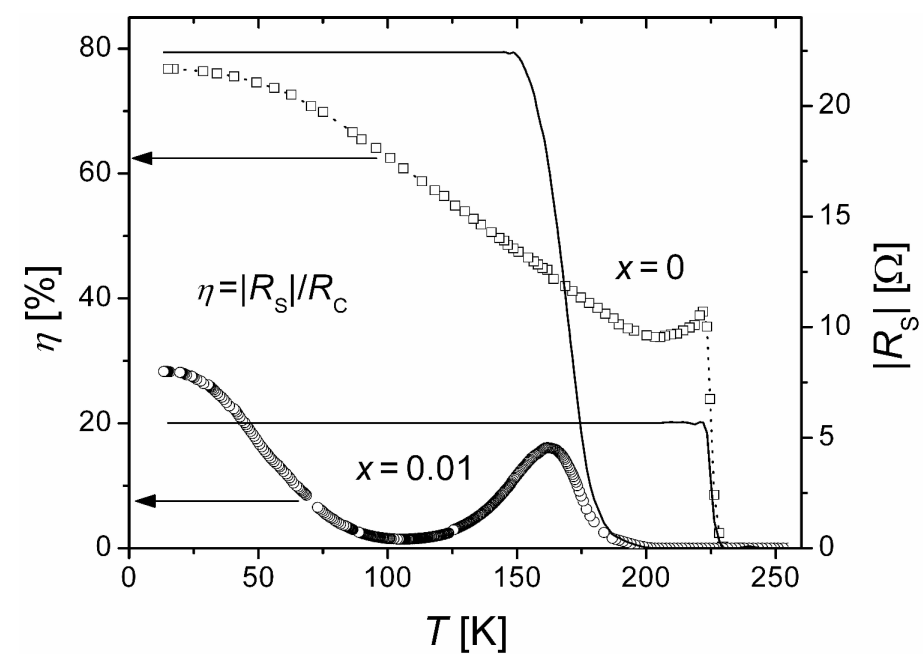

Fig. 7. Variation of resistance contribution $\left|R_{\mathrm{S}}\right|$ and "intrinsic" magnetoresistance $\eta$ with temperature for $\left(\mathrm{La}_{0.67} \mathrm{Ca}_{0.33}\right)\left(\mathrm{Mn}_{1-x} \mathrm{Sn}_{x}\right) \mathrm{O}_{3-\delta}$ compounds with $x=0$ and $x=0.01$. 
Such resistance changes observed near $T_{\mathrm{C}}$ are viewed as canonical for DE systems [21] and are intrinsic, bulk properties. Some authors call this colossal magnetoresistance effect observed in good-quality single crystals the "intrinsic" as opposed to the "extrinsic" magnetoresistance exhibited by the polycrystalline manganites, which is related to the presence of grain boundaries (GB) [22]. The "extrinsic" magnetoresistive effects are known to be due to spin-dependent scattering or tunnelling processes at the grain boundaries, inhomogeneities, microcracks and macroscopic defects. In the compounds with $x=0.01$ and $x=0.03$ this extrinsic part of the resistance plays a predominant role. Only in the $x=0$ compound both contributions are comparable. The temperature dependence of this "extrinsic" part of the resistance is also responsible for the broad maxima in the $R(T)$ curves observed below $T_{\mathrm{C}}$ in the $x=0,0.01$, and 0.03 compounds. The drops in the $R(T)$ values at $T_{\mathrm{C}}$ indicate that the $x=0$ and $x=0.01$ compounds are metallic inside the separated grains or micro-domains, but due to grain boundary effects they show insulating behaviour down to $T_{\mathrm{M}-\mathrm{I}}$. These results suggest a percolation nature of the $\mathrm{M}-\mathrm{I}$ transition in these compounds.

There exists some ambiguity in the literature concerning the dependence of the resistance on magnetization. It was reported to depend on $\left[1-\left(\frac{M}{M_{0}}\right)^{2}\right][23],\left[1-\left(\frac{M}{M_{0}}\right)\right][24], \exp \left[-\left(\frac{M}{M_{0}}\right)\right][25], \exp \left[-\left(\frac{M}{M_{0}}\right)^{2}\right][26]$, or $\exp \left\{-\left(\frac{T_{0}}{T}\right)\left[1-\left(\frac{M}{M_{0}}\right)^{2}\right]\right\}^{1 / 4}[20] . M_{0}$ is the saturation magnetization.

The $\left(\mathrm{La}_{0.67} \mathrm{Ca}_{0.33}\right)\left(\mathrm{Mn}_{1-x} \mathrm{Sn}_{x}\right) \mathrm{O}_{3-\delta}$ compounds with low $x$ values $(x \leq 0.01)$ - due to the relatively sharp magnetic transition at $T_{\mathrm{C}}$ and to the $\mathrm{Sn}$ adjustable separation between $T_{\mathrm{C}}$ and $T_{\mathrm{M}-\mathrm{I}}$ temperatures - offer a unique possibility to separate the $R_{\mathrm{S}}(T)$ contribution and to solve this problem. Precise measurements of the saturation magnetization $M_{0}(T)$ and of $R\left(M_{0}, T\right)$ are scheduled for these compounds. One should note that spontaneous magnetization is not the only source for the deviation from the straight line of the $\Delta R(T)$ curves in Fig. 5 , as was clearly shown by the shape of dashed curves for the $x=0$ and $x=0.01$ compounds. Both dashed curves show substantial deviations (but in opposite directions) from a straight line at $T_{\mathrm{C}}$. This shows clearly that the VRH model loses its applicability around $T_{\mathrm{C}}$ and that a new "extrinsic" mechanism starts to control the resistivity below $T_{\mathrm{C}}$.

The electrical resistance in the low temperature FM metallic region is usually fitted by a polynomial $R(T)=R_{0}+R_{\alpha} T^{\alpha}$, where $\alpha=2,2.5$, or $3, R_{0}$ is the residual resistance, $R_{\alpha}$ is the coefficient ascribed to electron-electron and electronphonon scattering but for temperatures higher than $\approx T_{\mathrm{C}} / 2$, an extra term $R_{\beta} T^{\beta}$ with $\beta$ larger than 3 is needed [1]. Such an approach seems to be most justified in the case of the $x=0$ compound, but even there the "extrinsic" part of the resistance seems to be predominant. We have proved that the polynomial $R(T)=$ $1.66(2)+1.658(1) \times 10^{-5} T^{2.5}$ fits best and in the largest temperature range (below 
$180 \mathrm{~K}$ ) experimental data for $x=0$ compound. It is worth noting that the experimental points between $50 \mathrm{~K}$ and $190 \mathrm{~K}$ can be fitted equally well by the exponential expression in the form $R(T)=1.01(1) \exp [0.0122(1) T]$. In the case of $x=0.01$ and $x=0.03$ compounds higher order polynomials have to be used to fit our data in reasonably extended temperature ranges in the low temperature ferromagnetic region.

As far as the specific heat results are concerned the anomalies for the $\left(\mathrm{La}_{0.67} \mathrm{Ca}_{0.33}\right)\left(\mathrm{Mn}_{1-x} \mathrm{Sn}_{x}\right) \mathrm{O}_{3-\delta}$ compounds with $x=0,0.01$, and 0.03 observed in the vicinity of the $T_{\mathrm{C}}$ show the onset of the magnetic ordering. No anomalies at $T_{\mathrm{M}-\mathrm{I}}$ transition temperatures are seen for the $x=0.01$ and $x=0$ compounds. For the $x=0$ compound a typical lambda anomaly with the largest magnetic entropy change is observed. Small Sn doping strongly diminishes the magnetic entropy at $T_{\mathrm{C}}$ by creating disorder in the Mn sublattice leading to hardly detectable effects for the $x=0.03$ compound.

\section{Conclusions}

Summarizing we conclude that:

1. High sintering temperature is needed to substitute $\mathrm{Sn}$ for $\mathrm{Mn}$ in $\left(\mathrm{La}_{0.67} \mathrm{Ca}_{0.33}\right) \mathrm{MnO}_{3-\delta}$ compound at levels $x \geq 0.01$ but this causes an oxygen deficiency of $\delta=0.06$. The large $\delta$-value shifts the compound close to the FM metal-FM insulator border and explains the appearance of the FM insulating state between the $T_{\mathrm{M}-\mathrm{I}}$ and $T_{\mathrm{C}}$ temperatures.

2. The introduction of a small amount of Sn has a great influence on the transport and magnetic properties of the parent compound due to combined effects of reducing the $\mathrm{DE}$ and introducing a local lattice distortions leading to a strong suppression of $T_{\mathrm{C}}$ and $T_{\mathrm{M}-\mathrm{I}}$.

3. All studied $\left(\mathrm{La}_{0.67} \mathrm{Ca}_{0.33}\right)\left(\mathrm{Mn}_{1-x} \mathrm{Sn}_{x}\right) \mathrm{O}_{3-\delta}$ compounds are paramagnetic insulators above the $T_{\mathrm{C}}$ temperatures and conduct via thermally assisted variable range hopping with the localization lengths $\xi$ equal to $2.2 \AA, 1.33 \AA$, and $1.26 \AA$ for $x=0,0.01$, and 0.03 , respectively.

4. The $x=0$ and $x=0.01$ compounds exhibited anomalies of $R(T)$ in accordance with the onset of the spontaneous magnetization $M_{\mathrm{S}}$ at corresponding $T_{\mathrm{C}}$. They offered a unique possibility to separate the contribution due to the spontaneous magnetization $\left(R_{\mathrm{S}}(T)\right)$ to the resistance and to estimate the magnitude of this "intrinsic" or spontaneous magnetoresistance effect (see Fig. 7).

5. In all $\left(\mathrm{La}_{0.67} \mathrm{Ca}_{0.33}\right)\left(\mathrm{Mn}_{1-x} \mathrm{Sn}_{x}\right) \mathrm{O}_{3-\delta}(x=0,0.01$, and 0.03) compounds the "extrinsic" part of the resistance which is related to the presence of grain boundaries plays a dominant role. This "extrinsic" part of the resistance is responsible for the broad maxima in the $R(T)$ curves observed below $T_{\mathrm{C}}$ 
in these compounds and defined as $T_{\mathrm{M}-\mathrm{I}}$. A percolation nature of the M-I transition in these compounds is suggested.

6. In the low temperature ferromagnetic region the polynomial $R(T)=$ $1.66(2)+1.658(1) \times 10^{-5} T^{2.5}$ (or exponential expression $R(T)=$ 1.01(1) $\exp [0.0122(1) T])$ fits best our resistance data for the $x=0$ compound. In the case of the $x=0.01$ and $x=0.03$ compounds higher order polynomials have to be used to fit our data in reasonably extended temperature ranges.

7. Small Sn doping strongly broadens and diminishes the specific heat jump at $T_{\mathrm{C}}$ most likely by creating disorder in the Mn sublattice leading to hardly detectable effects for the $x=0.03$ compound.

\section{Acknowledgments}

This work was supported by the Faculty of Physics and Nuclear Techniques, AGH University of Science and Technology, Cracow, through the statutory funds from the State Committee for Scientific Research (Poland). The support by the Austrian Exchange Service (ÖAD) Scientific \& Technological Cooperation Austria-Poland (project number $7 / 2003$ ) is appreciated.

\section{References}

[1] C.N.R. Rao, A.K. Raychaudhuri, in: Colossal Magnetoresistance, Charge Ordering and Related Properties of Manganese Oxides, Ed. C.N.R. Rao, B. Raveau, World Scientific, Singapore 1998, p. 1.

[2] R. Mahendiran, R. Mahesh, A.K. Raychaudhuri, C.N.R. Rao, Solid State Commun. 99, 149 (1996).

[3] R.D. Sánchez, J. Rivas, C. Vázquez-Vázquez, A. López-Quintela, M.T. Causa, M. Tovar, S. Oseroff, Appl. Phys. Lett. 68, 134 (1996).

[4] G.H. Rao, J.R. Sun, A. Kattwinkel, L. Haupt, K. Bärner, E. Schmitt, E. Gmelin, Physica B 269, 379 (1999).

[5] C. Zener, Phys. Rev. 82, 403 (1951).

[6] A.J. Millis, B.I. Shraiman, R. Mueller, Phys. Rev. Lett. 77, 175 (1996).

[7] E. Dagotto, T. Hotta, A. Moreo, Phys. Rep. 344, 1 (2001).

[8] G. Snyder, R. Hiskes, S. Di Carolis, M. Beasley, T. Geballe, Phys. Rev. B 53, 14434 (1996).

[9] N.F. Mott, J.H. Davis, Electronic Processes in Non-Crystalline Materials, 2nd ed., Oxford University Press, Oxford 1979.

[10] M.B. Salamon, M. Jaime, Rev. Mod Phys. 73, 583 (2001).

[11] A. Simopoulos, G. Kallias, E. Devlin, M. Pissas, Phys. Rev. B 63, 54403 (2000).

[12] A. Simopoulos, G. Kallias, E. Devlin, I. Panagiotopoulos, M. Pissas, J. Magn. Magn. Mater. 177-181, 860 (1998). 
[13] Z.W. Li, A.H. Morrish, J.Z. Jiang, Phys. Rev. B 60, 10284 (1999).

[14] L. Morales, A. Caneiro, R.D. Sánchez, D. Vega, J. Magn. Magn. Mater. 226-230, 806 (2001).

[15] L. Morales, R. Zysler, A. Caneiro, Physica B 320, 100 (2002).

[16] J. Rodriguez-Carvajal, Physica B 192, 55 (1993).

[17] J. Przewoźnik, J. Żukrowski, J. Chmist, E. Japa, A. Kołodziejczyk, K. Krop, K. Kellner, G. Gritzner, accepted for publication in Nukleonika, 2004.

[18] B. Dabrowski, R. Dybzinski, Z. Bukowski, O. Chmaissem, J.D. Jorgensen, J. Solid State Chem. 146, 448 (1999).

[19] S.-W. Choeng, H.Y. Hwang, in: Colossal Magnetoresistive Oxides, Ed. Y. Tokura, Gordon and Breach, London 2000, p. 237.

[20] M. Viret, L. Ranno, J.M.D. Coey, Phys. Rev. B 55, 8067 (1997).

[21] Y. Tokura, in Ref. [19], p. 1.

[22] B. Vertruyen, R. Cloots, A. Rulmont, G. Dhalenne, M. Ausloos, Ph. Vanderbemden, J. Appl. Phys. 90, 5692 (2001).

[23] Y.X. Jia, Li Lu, K. Khazeni, V.H. Crespi, A. Zettl, M.L. Cohen, Phys. Rev. B 52, 9147 (1995).

[24] P.S. Anil Kumar, P.A. Joy, S.K. Date, J. Phys., Condens. Matter 10, L269 (1998).

[25] M.F. Hundley, M. Hawley, R.H. Heffner, Q.X. Jia, J.J. Neumeier, J. Tesmer, J.D. Thompson, X.D. Wu, Appl. Phys. Lett. 67, 860 (1995).

[26] J. O'Donnell, M. Onellion, M.S. Rzchowski, J.N. Eckstein, I. Bozovic, Phys. Rev. B 54, R6841 (1996). 